\title{
Le grand oublié des réformes éducatives
}

L'enseignement primaire en Espagne

Falling by the School Reform Wayside: Spanish Primary Education

La gran olvidada de las reformas escolares : la enseñanza primaria española

\section{Juan Carlos González Faraco et Antonio Luzón Trujillo}

Traducteur : Anne Jumelet

\section{(2) OpenEdition}

\section{Journals}

Édition électronique

URL : http://journals.openedition.org/ries/1146

DOI : 10.4000/ries. 1146

ISSN : 2261-4265

Éditeur

Centre international d'études pédagogiques

Édition imprimée

Date de publication : 1 avril 2006

Pagination : $25-40$

ISSN : $1254-4590$

Référence électronique

Juan Carlos González Faraco et Antonio Luzón Trujillo, "Le grand oublié des réformes éducatives », Revue internationale d'éducation de Sèvres [En ligne], 41 | avril 2006, mis en ligne le 15 novembre 2011, consulté le 01 mai 2019. URL : http://journals.openedition.org/ries/1146 ; DOI : 10.4000/ries.1146 


\title{
Le grand oublié des réformes éducatives*
}

\section{L'enseignement primaire en Espagne}

\author{
Juan Carlos Gonzalez Faraco \\ Antonio Luzon Trujillo
}

L'enseignement primaire a été conçu, à l'origine des systèmes scolaires modernes, comme la base d'une pyramide dont le sommet n'était accessible qu'à une minorité privilégiée. Selon ce schéma, l'enseignement secondaire avait la tâche ingrate de procéder à la sélection des élèves. Au cours de la seconde moitié du XX $\mathrm{XX}^{\mathrm{e}}$ siècle, ce modèle s'est progressivement et profondément dégradé, à un rythme différent selon les cas. Les nouvelles politiques des pays les plus développés ont mis l'accent sur la démocratisation et l'équité, ce qui a déclenché un flot ininterrompu de réformes scolaires qui, par ailleurs, ont été freinées ou réorientées dans les dernières décennies du siècle par des politiques néolibérales. La demande éducative a pris une extraordinaire importance, la scolarisation à l'école primaire a touché l'ensemble de la population enfantine et la massification de l'enseignement secondaire est devenue une réalité (Ramírez et Ventresca, 1992). En toute logique, dans ce contexte nouveau et dynamique, le rôle éducatif et social de l'enseignement primaire a été profondément modifié.

Dans le cadre européen, le cas espagnol est d'une certaine façon particulier à cause du retard pris par ces phénomènes et de l'amoncellement de réformes en un laps de temps relativement court. Cela s'explique par l'histoire politique du pays (une longue dictature, un régime démocratique encore jeune). En tout état de cause, le système scolaire espagnol, et en particulier l'enseignement primaire, ont connu et continuent à connaître depuis trente ans, une série de réformes marquées du sceau du désaccord, de la polémique et par conséquent de la fugacité (De Puelles, 2005). Il faut ajouter que, pendant cette période, l'enseignement primaire, selon l'opinion communément répandue, a perdu le premier rôle pour devenir injustement et inexplicablement le grand oublié de la politique éducative (Lledó, 1999; Doménech, 2005).

Dans cet article, on s'interrogera d'une part sur le comment et le pourquoi de ce processus de relégation et même de dévalorisation, et d'autre part on indiquera ce que représentait auparavant et ce que représente maintenant l'enseignement primaire sur le plan pédagogique. Mais avant tout, on cherchera

\footnotetext{
* Article traduit par Anne Jumelet.
} 
à cerner les problèmes et suggérer quels sont les défis qui se posent à l'école primaire espagnole derrière le calme apparent, défis en partie différents mais aussi, dans une grande mesure, très semblables à ceux auxquels sont confrontés d'autres systèmes scolaires.

Traditionnellement en Espagne, les termes "école primaire», "école de base » et «école obligatoire» étaient synonymes jusqu'à la réforme initiée par le gouvernement socialiste en 1990. Dans cette réforme, la scolarité obligatoire jusqu'à quatorze ans a été prolongée jusqu'à seize ans et divisée en deux étapes : l'enseignement primaire et l'enseignement secondaire obligatoire. Depuis lors et jusqu'à présent, l'école primaire est le premier échelon de l'école de base, comme c'est d'ailleurs le cas dans d'autres systèmes européens (Green, Leney et Wolf, 2001).

Cependant, la transformation majeure qu'a connue l'école primaire s'est produite vingt ans plus tôt, en 1970, dans le cadre d'une ambitieuse réforme scolaire promue par l'un des derniers gouvernements du général Franco. Cette réforme a abandonné le modèle de référence du système éducatif espagnol depuis ses origines - le modèle français - pour un modèle inspiré des systèmes scandinaves. L'objectif était de donner au système éducatif espagnol une dimension plus polyvalente, donc plus égalitaire. En conséquence, l'enseignement primaire a changé de structure, de sens et même de nom. On l'a appelé enseignement général de base.

\section{L'ENSEIGNEMENT GÉNÉRAL DE BASE}

Il ne faut pas oublier que, jusqu'en 1970, les élèves étaient triés à l'âge de dix ans, c'est-à-dire après avoir partagé seulement quatre années d'enseignement. Une partie d'entre eux, après avoir réussi l'examen national, entrait dans le secondaire (baccalauréat élémentaire), tandis que les autres, les plus nombreux, devaient se contenter, dans le meilleur des cas, de poursuivre leur scolarité à l'école primaire jusqu'à quatorze ans avant de trouver un emploi le plus souvent peu qualifié. Ce tri prématuré introduisait une discrimination entre les élèves, sans compter les effets très négatifs sur l'intégration sociale dans le pays (Viñao, 1992; Pérez Díaz et Rodríguez, 2003). Il y avait donc un système de scolarisation double, avec deux enseignements de base parallèles et étanches.

La réforme de 1970 a tenté de produire un réel effort de modernisation de l'éducation d'une nation dont le niveau de développement économique et social contrastait de plus en plus avec la dictature politique encore en vigueur. Cette réforme devait servir, entre autres choses, à adoucir cette contradiction flagrante et à donner une légitimité, dans la mesure du possible, à un régime politique de toute évidence dépassé. Par ailleurs, il est sûr que cette réforme se faisait aussi l'écho de deux valeurs sociales émergentes en Espagne au début des années soixante : une plus grande sensibilité à l'égalité des chances ainsi que le 
désir de réussite et de mobilité sociale grâce à l'effort et au mérite individuel. Le système scolaire est devenu alors le catalyseur d'une grande partie des conflits et des aspirations de la société et la demande d'éducation a connu une croissance sans précédent.

Les conséquences de la réforme de 1970 pour l'enseignement primaire ont été très importantes. Un nouveau modèle généraliste, articulé autour d'un seul et unique cycle commun pour tous les enfants soumis à l'obligation scolaire, a vu le jour (Viñao, 1992). Cette nouvelle conception de l'enseignement primaire a occasionné de multiples changements. La structure, les programmes, le corps professoral et, bien évidemment, le vocabulaire ont connu de substantielles transformations. Comme nous l'avons déjà dit, la traditionnelle dénomination "primaire» a cédé la place à «enseignement général de base» (EGB) et les maîtres ont été appelés "professeurs d'EGB».

Il est vrai qu'en adoptant une nouvelle terminologie, les réformes éducatives ambitionnent de donner l'impression, et parfois même l'illusion, d'une transformation réelle et d'une rupture avec le passé (Bolívar et Rodríguez Diéguez, 2002). Dans le cas présent, les changements lexicaux n'ont pas été pure rhétorique, même si certaines de ces réformes étaient déjà en gestation tandis que d'autres n'ont jamais vu le jour. Il est sûr que l'enseignement primaire traditionnel a connu là une véritable revalorisation s'inscrivant comme une étape fondamentale de la nouvelle structure du système éducatif espagnol. On peut même dire que l'enseignement primaire a été la vedette de cette réforme. C'était normal puisque l'Espagne traînait derrière elle une longue histoire de précarité éducative, avec des taux de scolarisation qui, s'ils s'étaient considérablement améliorés durant la décennie précédente, étaient encore en dessous de la moyenne européenne. En 1970, il subsistait un déficit d'un million de places à l'école et la scolarité obligatoire n'était pas complètement assurée par l'État (Pérez Díaz et Rodríguez, 2003).

Pour la première fois de leur histoire, les Espagnols ont bénéficié d'une école de base unifiée, commune et gratuite d'une durée de huit ans, dispensée dans des établissements spécifiques par des professeurs dont la formation initiale se faisait désormais à l'université. Les anciennes Écoles normales de maîtres, à la faible réputation académique, sont devenues des Écoles universitaires de formation des professeurs d'EGB. Au début, ces changements d'appellation n'ont pas réellement modifié le statut ni les caractéristiques des institutions et du corps professoral, mais ils étaient hautement symboliques. Le mot «maître», si agréable à entendre, était cependant associé à un passé marqué par une formation insuffisante, de bas salaires et le manque de prestige de la profession. L'enseignement primaire souffrait de maux semblables, puisque l'ensemble du système scolaire espagnol avait été traditionnellement conçu à partir de son sommet et non de sa base. 
La réforme de 1970 - ce qui constituait une autre nouveauté - a tenté de faire évoluer cette conception de classe élitiste, et a concentré une bonne partie de ses efforts sur l'amélioration radicale de cette étape du système. D'une part, la création de l'enseignement général de base a favorisé, avec de nombreux autres facteurs socio-économiques et culturels, la tendance à l'augmentation progressive des taux de scolarisation. D'autre part, la disparition du «baccalauréat élémentaire», qui avait servi d'outil de sélection précoce, a amélioré l'équité du système scolaire.

L'influence des sciences de l'éducation a été un autre facteur décisif de cette réforme en inondant les écoles d'un langage nouveau. Le terme d' "année ", dont la référence était strictement quantitative, a été remplacé par celui de «niveau», concept beaucoup plus éducatif, lié au rythme d'apprentissage des élèves. L'enseignement général de base a été divisé en huit niveaux répartis en deux étapes, la première comportant une approche intégrée et globale des savoirs et la deuxième s'appuyant davantage sur les disciplines, ce qui a entraîné la création de spécialités d'enseignement chez les professeurs. Quelques années plus tard, l'EGB a été divisé en trois cycles (initial, moyen et supérieur). Avec ces cycles, on souhaitait limiter la fragmentation excessive du temps scolaire et favoriser ainsi l'individualisation de l'apprentissage, le travail en équipe chez les professeurs, le contrôle continu, et enfin, la réduction de l'échec scolaire. À la fin de l'EGB, les élèves obtenaient le «certificat scolaire», le premier diplôme offert alors par le système scolaire espagnol.

Les programmes ont été profondément remaniés par des «orientations pédagogiques » successives, documents nationaux qui se faisaient l'écho de l'esprit de la «nouvelle École» et de la pédagogie active, au moins dans sa dimension didactique. Naturellement, les nouvelles exigences curriculaires ont rencontré de sérieuses résistances dans une grammaire scolaire marquée par des habitudes et des méthodes très traditionnelles : primauté du livre de textes, épreuves conventionnelles, organisation disciplinaire du curriculum, etc., pratiques étrangères au concept d'école de base, mais typiques du style éducatif de l'enseignement secondaire.

\section{LA RÉFORME SOCIALISTE DES ANNÉES 90 : ÉCOLE PRIMAIRE VS ÉCOLE DE BASE}

La grande réforme suivante du système scolaire espagnol, comme nous l'avons déjà dit, a eu lieu en 1990 et a été impulsée par le premier gouvernement socialiste. On la connaît en Espagne sous le sigle de la loi qui l'a régulée sur le plan juridique : LOGSE (Loi d'organisation générale du système éducatif). Pendant les vingt années qui séparent cette loi de la précédente, la vie politique espagnole a connu de grands changements : la disparition de la dictature franquiste 
et l'avènement d'un régime démocratique, la décentralisation de l'État et la création de communautés autonomes, l'entrée de l'Espagne dans l'Union européenne et, enfin, l'accélération du processus de développement économique et de modernisation sociale du pays.

Sur le plan éducatif, les résultats produits par la réforme de 1970 et ses modifications ultérieures n'ont pas été aussi satisfaisants que prévu. Le taux de scolarisation s'est amélioré, mais l'offre de places à l'école s'est révélée insuffisante pour accueillir dans de bonnes conditions une demande croissante. L'école publique a été la plus touchée par ces insuffisances. La situation ne s'est pas améliorée avant les années quatre-vingt, avec l'adoption de mesures comme la construction d'établissements scolaires, de nouvelles orientations pédagogiques, des programmes de soutien aux élèves handicapés, et la mise en œuvre de programmes d'éducation compensatoire dans les zones socialement défavorisées. Dans ce domaine, les recherches étaient unanimes : le degré d'équité du système était toujours très insuffisant et les effets de l'origine sociale sur les inégalités éducatives toujours aussi déterminants.

\section{Une division de la scolarité de base : le primaire comme étape intermédiaire}

Dans ce contexte, la réforme socialiste de 1990 a tenté d'approfondir et de développer le principe clé de la réforme antérieure, l'idéal d'une école pour tous, mais en adoptant, structurellement parlant, une stratégie inverse, c'est-àdire en réduisant la durée de l'enseignement général de base (qui a retrouvé son nom d'enseignement primaire) et en recréant l'étape secondaire inférieure (qui a pris le nom d'enseignement secondaire obligatoire). Ce schéma structurel a survécu à deux nouvelles réformes générales du système espagnol. Depuis lors, le primaire est une étape relativement homogène, pleinement polyvalente, d'une durée de six années, accueillant les élèves âgés de six à douze ans, divisée en trois cycles successifs de deux ans. L'enseignement est dispensé dans des établissements spécifiques, qui dans le secteur public reçoivent généralement aussi le deuxième cycle de l'enseignement pré-élémentaire (en Espagne, enseignement enfantin) : les «centres d'enseignement enfantin et primaire». Ce modèle est assez rare en Europe où il est plus fréquent que l'enseignement préprimaire dispose de ses propres établissements.

L'aménagement des transitions est, on le sait, d'une importance capitale pour la structuration des systèmes scolaires et le développement éducatif des élèves. Dans le cas espagnol, la transition entre les étapes préprimaire et primaire est peu marquée, ce qui est facilité par le fait, entre autres, que le corps professoral est le même et peut passer facilement d'une étape à l'autre. La situation est différente entre l'enseignement primaire et l'enseignement secondaire inférieur. En général, sauf dans certaines zones rurales, les élèves, à la fin du 
primaire, vont dans un institut d'enseignement secondaire (IES), pour suivre les cours de l'enseignement secondaire inférieur et aussi le plus souvent l'enseignement secondaire supérieur (baccalauréat). Alors que l'enseignement préprimaire peut subir l'influence de la culture scolaire de l'enseignement primaire et perdre en partie ses spécificités psychopédagogiques, l'enseignement secondaire inférieur est clairement marqué par la culture enseignante, profondément attachée aux disciplines, de l'enseignement secondaire supérieur. Ce qui, ajouté à d'autres discordances, rend plus difficile la transition entre l'école primaire et l'enseignement secondaire inférieur bien que tous deux fassent partie de la scolarité de base.

Si l'enseignement primaire a été, comme nous l'avons dit, la vedette de la réforme de 1970, depuis la réforme de 1990 c'est le tour de l'enseignement secondaire obligatoire (ESO). C'est sans doute parce que celui-ci symbolise un des grands objectifs de la réforme socialiste (étendre massivement la scolarisation dans le secondaire et par conséquent le caractère polyvalent de l'école de base) mais aussi parce que c'est là que se concentrent les problèmes de discipline et de rendement scolaire les plus importants. L'ESO est donc devenu le grand défi des politiques éducatives et des analyses pédagogiques. À l'inverse, l'enseignement primaire étant placé à cheval entre l'enseignement de base et l'enseignement secondaire et ayant perdu son caractère terminal (il ne délivre plus de diplôme propre), est relégué au second plan. Le primaire est probablement de plus en plus souvent perçu, face au «chaos» du secondaire, comme une étape éducative consolidée et tranquille, dont le public présente un profil moins problématique. Cette perception, fausse en grande partie, oublie que bien des problèmes scolaires qui émergent dans le secondaire ont leurs racines au stade du primaire, en particulier tout ce qui concerne les inégalités, l'échec et les difficultés d'adaptation des élèves.

\section{La primauté de la méthode sur les contenus}

Selon l'article 12 de la LOGSE, l'enseignement primaire a pour principaux objectifs de «fournir à tous les enfants une éducation commune qui rende possible l'acquisition d'une culture de base, des apprentissages relatifs à l'expression orale, à la lecture, à l'écriture et au calcul, ainsi qu'une progressive autonomie à agir dans son milieu».

Le plan curriculaire dessiné par cette réforme, qui proclame son allégeance sur le plan théorique au constructivisme, a été organisé autour de trois catégories de contenus ou domaines d'apprentissage : les concepts (référés à des faits et à des principes), les procédés (en lien avec le savoir faire et la façon d'acquérir de nouvelles connaissances) et les attitudes (normes et valeurs). Cette conception globale et exhaustive de la connaissance humaine implique des changements tant pour la planification de l'enseignement et le développement de l'apprentissage que pour l'évaluation. Cette dernière, comme c'était le cas 
pour l'enseignement général de base, devrait être «continue et globale». À la fin de chaque cycle, le maître-tuteur de chaque groupe classe décide du passage de ses élèves dans le cycle supérieur s'ils ont atteint les objectifs prévus, et utilise pour cela des formules qualitatives telles que "progrès convenables» ou «doit progresser». En théorie, le caractère global de l'évaluation implique également l'autoévaluation de sa propre pratique, ce qui arrive rarement.

Les contenus des enseignements se répartissent, à l'instar de la réforme antérieure, en domaines de connaissance. Ce sont : la langue espagnole (et, le cas échéant, la langue officielle de la communauté autonome), la connaissance du milieu naturel, social et culturel, les mathématiques, une langue vivante étrangère, l'éducation artistique et l'éducation physique. Une mention particulière est faite de l'enseignement de la religion catholique, en raison de l'incessante polémique sur sa régulation dans les établissements scolaires. Bien que l'État espagnol soit non confessionnel, en vertu des accords passés avec le Saint Siège, tous les établissements publics doivent offrir à leurs élèves la possibilité de choisir cette matière comme option.

La réforme des années quatre-vingt dix a déclenché elle aussi une véritable avalanche de termes psychopédagogiques, pour la plupart étrangers à la culture professionnelle des maîtres : "apprentissage significatif», "projet curriculaire», "projet d'établissement», "plan curriculaire de base», "adaptation curriculaire», etc. Grâce à la création d'un réseau local de centres pour la formation continue des professeurs (CEPs), les autorités éducatives ont voulu inoculer ce vocabulaire novateur aux maîtres et les gagner à la cause de la réforme. Mais les résistances au changement de modèle pédagogique se sont manifestées dès le départ. Pour de nombreux professeurs, il s'agissait seulement d'un «jargon» purement formel, éloigné de la réalité des écoles.

Le scepticisme des professeurs n'était qu'en partie justifié. Les résultats obtenus par les élèves de l'enseignement primaire aux évaluations nationales (1995 et 1999), sans être tout à fait satisfaisants, ont été meilleurs que ceux obtenus par les élèves de l'enseignement général de base. Cependant, le primaire n'est pas parvenu à se défaire de certains des «vices » typiques de l'EGB, comme par exemple, le manque de coordination entre les niveaux et les cycles, ce qui «balkanise» l'enseignement dans les établissements et fait des classes des compartiments étanches; ou par exemple, la faible relation entre les domaines de connaissance et les spécialités, ce qui est en contradiction avec l'orientation globale et interdisciplinaire donnée à une école véritablement polyvalente et amoindrit la cohérence des programmes. Ce manque de coordination est encore plus important, comme cela a déjà été souligné, entre l'enseignement primaire et les étapes voisines, l'enseignement pré-élémentaire et l'enseignement secondaire, ce qui de toute évidence provoque l'isolement de l'enseignement primaire (Rogero, 1997 ; Pérez Díaz et Rodríguez, 2003). 


\section{Les effets \\ de la démocratisation et de la décentralisation}

Le processus de décentralisation politique issu de la constitution de 1978 est un des facteurs essentiels pour comprendre l'évolution de l'enseignement primaire en Espagne. L'État se réserve l'orientation des grandes lignes de la politique éducative, mais ce sont les gouvernements des communautés autonomes qui planifient, dirigent et administrent le système scolaire sur leur territoire. Ce sont eux et non les autorités locales ou municipales qui détiennent le pouvoir scolaire, ce qui donne un centralisme régional très accusé.

C'est le gouvernement central qui est compétent pour définir les aspects essentiels du curriculum, c'est-à-dire les enseignements minimums communs à tout le territoire national. Les gouvernements autonomes élaborent les bases des programmes officiels pour les établissements de leur territoire à partir de ce cadre national: les objectifs généraux pour chaque étape, les contenus minimums et quelques orientations méthodologiques et d'évaluation. En conséquence, on note des différences entre les communautés quant aux contenus et à leur organisation, au nombre d'heures d'enseignement affectées à chaque domaine de connaissance et à d'autres aspects fondamentaux de l'enseignement primaire. Il n'est pas exagéré d'affirmer que celui-ci a perdu son caractère national et unifié, même s'il s'avère que les différences de rendement et le niveau des inégalités sont surtout liés au type d'établissement (public, privé sous contrat, privé hors contrat) et à l'origine sociale des élèves, ou à tous ces facteurs en même temps. Ce n'est pas un hasard si les communautés autonomes espagnoles qui connaissent les meilleurs résultats aux évaluations internationales sont celles où l'école privée est la mieux implantée dans le primaire et le secondaire.

Pour en revenir aux programmes, il faut savoir que les instructions données par le ministère de l'Éducation sont fixées par un texte appelé «plan curriculaire de base», qui a un caractère ouvert et flexible et qui doit donc être peu à peu mis en œuvre et adapté, dans un premier temps par les administrations régionales autonomes, ensuite par les établissements («projet curriculaire d'établissement») et enfin par les maîtres dans leur classe ("projet curriculaire de classe»). Ce sont donc eux qui sont les véritables interprètes des fonctions sociales et éducatives que la société assigne aux écoles primaires. Dans un contexte d'autonomie scolaire comme celui que connaît l'Espagne, les maîtres disposent d'une vaste marge d'action, propice à l'introduction d'innovations pédagogiques et à l'amélioration de leurs pratiques. Ces possibilités se heurtent souvent à un habitus professionnel complaisant et routinier, ce qui n'est pas étranger à la condition de fonctionnaire de la majorité des maîtres en Espagne. 


\section{LA DIALECTIQUE ENTRE LA TRADITION ET L'INNOVATION}

Nous avons vu comment dans les trois dernières décennies, l'enseignement primaire a cessé d'être une priorité pour la politique éducative espagnole et un objet valorisé par une bonne partie de la population, pour se transformer en étape de passage vers le secondaire, qui actuellement concentre toute l'attention de l'institution et de la société. La cascade de réformes élaborées dans les cinq dernières années n'a pas infléchi cette tendance à l'oubli de l'enseignement primaire. Il s'agit de la Loi de qualité de l'éducation, approuvée en 2002 par le gouvernement conservateur du Parti populaire et de la Loi organique de l'éducation, promulguée sous l'égide du gouvernement socialiste en 2005. La première d'entre elles est à peine entrée en application, mais elle était focalisée de toute façon sur le secondaire inférieur, dont le deuxième cycle introduisait des itinéraires parallèles en fonction des centres d'intérêt et des résultats des élèves. À peine parvenus au pouvoir en 2004, les socialistes entament leur propre processus de réforme, qui suspend la réforme conservatrice et reprend les principes essentiels de leur réforme de 1990.

Cette vertigineuse succession de réformes et de contre-réformes et le manque de consensus social à leur propos, hélas traditionnel dans le secteur éducatif en Espagne, maintiennent le système scolaire dans un état d'incertitude permanente et provoquent inévitablement désenchantement, fatigue et sentiment d'impuissance (De Puelles, 2005). À ce rythme, les réformes sont régulièrement condamnées à l'échec, essentiellement parce que, en raison de leur nature même, elles exigent un temps long pour produire les effets recherchés. À ce petit jeu qui consiste, telle Pénélope, à tisser et à défaire ce que l'on a tissé, quel est le rôle de l'enseignement primaire? Quels changements a-t-il connu? Reste-t-il figé et immobile à l'abri du «tsunami» de réformes qui a affligé le système éducatif espagnol au cours des cinq premières années du XXI ${ }^{\mathrm{e}}$ siècle? En définitive, quels sont aujourd'hui le profil et les perspectives d'avenir de l'école primaire en Espagne?

\section{UN CURRICULUM EN CONSTRUCTION}

Bien qu'il se soit écoulé quinze années depuis que l'enseignement primaire a été configuré comme tel en Espagne, il est toujours considéré comme une étape préparatoire au secondaire. L'article 15 de la Loi de qualité le confirme très clairement. "La finalité de l'enseignement primaire est de faciliter l'apprentissage de l'expression et de la compréhension orale, de la lecture, de l'écriture, du calcul, ainsi que l'acquisition d'un socle culturel et d'habitudes de vie en collectivité, de travail et d'effort, afin de garantir une formation complète qui contribue à l'épanouissement de la personnalité et prépare les 
élèves à poursuivre avec profit leur scolarité dans l'enseignement secondaire obligatoire». Ce caractère propédeutique amoindrit sans aucun doute son identité propre, son autonomie et sa substance.

\section{Compétences de base}

Il reste donc nécessaire et urgent de retrouver et de redéfinir un espace curriculaire propre au sein de la scolarité de base (LLedó, 1999). L'enseignement primaire est toujours principalement associé à l'alphabétisation traditionnelle, c'est-à-dire aux apprentissages instrumentaux (lecture, écriture et calculs) qui l'emportent largement sur l'apprentissage d'habiletés (dans le sens «d'apprendre à apprendre", de manier d'autres langages, etc.) et sur les objectifs à caractère social et axiologique. La nouvelle loi socialiste tente de minimiser cette primauté, si enracinée dans la culture enseignante, en adoptant le concept de compétences de base, pour donner à l'apprentissage une dimension plus exhaustive.

\section{Éducation aux valeurs}

Pourtant, le débat actuel sur les programmes de l'enseignement primaire espagnol dégage deux priorités : mettre en œuvre son orientation axiologique et étendre l'utilisation des nouvelles technologies. Pour la première, il est prévu d'introduire une nouvelle discipline dans le dernier cycle (10-12 ans), "l'éducation à la citoyenneté", dans le cadre de «l'éducation aux valeurs», un ensemble diffus de matières transversales, vantées comme grande innovation par la réforme des années quatre-vingt dix mais dont l'application s'est révélée généralement précaire (González Faraco, 1999). Par ailleurs, «l'éducation à la citoyenneté» est née à la suite de l'affrontement à propos de l'enseignement de la religion entre la hiérarchie ecclésiastique catholique et le gouvernement socialiste, qui tente de réduire l'importance de cette discipline dans le système scolaire public. "L'éducation à la citoyenneté", qui aura un caractère général et obligatoire, traitera de la formation civique et éthique des élèves, c'est-à-dire de l'éducation aux valeurs morales, une nécessité revendiquée par l'Église de manière réitérée pour défendre la pertinence de la formation religieuse.

\section{Nouvelles technologies}

Les nouvelles technologies d'information et de communication (NTIC) sont, pour beaucoup, une voie prometteuse de pénétration des innovations didactiques et de renouveau de l'école. De fait, la maîtrise des NTIC est probablement l'apprentissage le plus demandé par les maîtres en formation continue et le plus favorisé par les programmes officiels d'amélioration du système éducatif. Les "nouvelles technologies" sont sans aucun doute les nouvelles vedettes de la politique éducative et pour bien des acteurs une véritable «mystique» qui va révolutionner l'école publique. 
Ainsi, alors que la substantifique moelle du curriculum demeure inchangée, le pari de l'innovation se concentre presque exclusivement, et parfois presque de manière obsessionnelle, sur le changement technologique, et sur quelques autres mesures comme l'apprentissage des langues étrangères, un domaine où les résultats des élèves espagnols se révèlent dramatiquement insuffisants. Pour cet apprentissage, l'innovation se limite à avancer le démarrage de la première langue (en général l'anglais) en dernière année de l'enseignement préélémentaire, et à introduire une seconde langue (le plus souvent le français) au troisième cycle du primaire. Il n'est en aucun cas envisagé d'améliorer la qualité méthodologique de l'enseignement, ni la formation des professeurs, pas plus que de réduire la taille des groupes pour enseigner cette discipline.

\section{Diversité et inégalité à l'école primaire}

En dehors de la nouveauté que peut représenter «l'éducation à la citoyenneté», il ne semble pas que la réforme en cours modifie substantiellement le positionnement de l'enseignement primaire comme étape de passage (Feito, 2005). Cependant, il convient de signaler un ensemble de mesures qui ont pour objet de corriger certains des problèmes déjà soulevés, pour l'essentiel ceux qui concernent sa capacité à combattre les inégalités et l'exclusion.

Étant donné les évidentes insuffisances du système éducatif espagnol dans ce domaine (Pereyra, González Faraco et Torres, 2005), bon nombre de recherches issues de méthodologies et d'origines disciplinaires variées se sont interrogées de manière réitérée sur les effets réels de la rhétorique de la "rédemption sociale» qui a accompagné la réforme socialiste des années quatre-vingt dix (Rambla et Bonal, 2000; Sevilla, 2003). On ne s'étonnera donc pas de ce que la nouvelle loi socialiste souligne avec emphase, à propos de l'enseignement primaire, le principe pédagogique de l'accueil de la diversité, ce qui suppose de reconnaître que c'est à l'école primaire que naissent les inégalités et que c'est à elle de les prévenir.

La loi propose par conséquent des mesures de soutien, dès que les premières difficultés d'apprentissage apparaissent, ainsi que des mesures de rattrapage des compétences de base qui n'ont pas été acquises. Selon le rapport d'évaluation de l'institut national de qualité et d'évaluation de 1995, $49 \%$ et $26 \%$ des élèves de douze ans n'ont pas eu de résultats suffisants respectivement en mathématiques et en langue espagnole. En 1998, un nouveau rapport du même organisme affirme que $25 \%$ des élèves, à la fin du primaire, ont toujours des résultats insuffisants dans ces apprentissages (ministère de l'éducation, 1998, p. 145). La loi socialiste actuelle reconnaît elle aussi ouvertement ce problème.

Ainsi donc, la nécessité de doter le système d'instruments qui préviennent et luttent précocement contre l'échec, qui favorisent l'égalité et permettent l'intégration de tous les élèves, revient au premier plan de la politique 
éducative. La présence croissante d'enfants issus de familles immigrées dans le système scolaire espagnol représente, pour les politiques d'accueil de la diversité, un nouveau domaine d'action tout à fait prioritaire.

Le multiculturalisme à l'école est un phénomène récent en Espagne, à la portée statistique encore limitée si on la compare à celle d'autres pays européens, mais qui augmente et se diversifie rapidement. Le nombre d'élèves étrangers scolarisés a été décuplé ces dix dernières années (à l'heure actuelle, il se monte en valeur absolue à cinq cent mille). Aujourd'hui, il s'élève au niveau national à $6,5 \%$ des élèves de l'enseignement non universitaire, chiffre qui atteint $11 \%$ à Madrid. En primaire, la moyenne est de $8 \%$ (dans le secteur public, elle atteint $10 \%$ ) ce qui représente au total deux cent mille élèves. Leur répartition sur le territoire espagnol est très inégale et variable quant au volume, à l'origine culturelle, à la maîtrise linguistique, à la stabilité professionnelle des parents, etc., ce qui donne des situations très diverses qui nécessitent naturellement des programmes éducatifs différenciés et flexibles.

La pratique effective du contrôle continu - un vieil objectif qui n'est jamais vraiment appliqué - devrait permettre aux établissements d'inclure dans leurs projets éducatifs des initiatives spécifiques pour les élèves qui rencontrent des difficultés d'apprentissage, quelle qu'en soit l'origine. La nouvelle réforme insiste sur des voies déjà connues comme les «adaptations curriculaires individualisées» pour les élèves à besoins éducatifs particuliers, et la "diversification curriculaire» pour aborder les retards dans l'apprentissage de certains groupes d'élèves. D'autres mesures viennent s'ajouter, difficiles à mettre en ouvre en raison de leur coût et des résistances opposées par l'organisation rigide des écoles : des regroupements flexibles d'élèves, avec l'utilisation de groupes parallèles pour les apprentissages instrumentaux de base; davantage d'heures de travail au sein de l'établissement; des activités scolaires complémentaires; un plan spécial de soutien pendant l'été; le maintien de l'élève un an de plus dans le même cycle, etc.

À la fin de la quatrième année de primaire, c'est-à-dire du deuxième cycle, on propose une "évaluation diagnostic» menée par les établissements, initiative qui existait déjà dans la Loi de qualité de 2002. Cette épreuve servira en principe à l'information en interne et à la planification, par les établissements eux-mêmes et par les administrations autonomes, de l'aide et du soutien nécessaires. Mais il est à craindre qu'elle ne devienne finalement un système d'évaluation publique établissant un classement des écoles. Le primaire participerait de cette façon aux processus de mesure, de standardisation et de compétition que connaît déjà le secondaire, objet habituel d'évaluations internationales comme celles que mène régulièrement l'OCDE. Les effets de ces évaluations sur l'autonomie scolaire et sur l'égalité des chances dans l'école de base peuvent être ravageurs (Feito, 2005). 


\section{RÉINVENTER L'ENSEIGNEMENT PRIMAIRE}

Il a été dit avec insistance que l'enseignement primaire a cessé d'être un objectif stratégique pour devenir une simple étape de passage et, dans une certaine mesure, une étape accessoire du cycle de réformes vécu par le système éducatif espagnol depuis 1970. Les trois dernières grandes réformes (1990, 2002 et 2005), d'orientations politiques diverses, ont toutes contribué à cette relégation et à cet oubli. Le primaire a cessé d'être une priorité politique au bénéfice de l'enseignement secondaire obligatoire, avec lequel il se partage la scolarité de base. Son autonomie et son identité pédagogique ont été dévalorisées lorsqu'il a été considéré comme une étape intermédiaire, transitoire, préparatoire et qu'il a par conséquent été marqué, surtout pour le cycle final, par le style éducatif fondé sur les disciplines de l'enseignement secondaire. La nouvelle réforme, en plein débat aujourd'hui, n'aborde pas directement ce problème de fond; elle se limite à introduire quelques retouches curriculaires mineures et à créer des programmes attractifs qui donnent, le plus souvent avec des moyens insuffisants, une image moderne et innovante de l'éducation espagnole : nouvelles technologies, formation plurilingue, co-éducation, dimension européenne...

Il est possible que l'enseignement primaire soit prisonnier d'une fausse image ou, tout au moins, d'une image biaisée qui s'est peu à peu formée à son sujet. Tout d'abord, l'idée que l'école primaire est encore attachée au passé et tend à l'immobilisme est pour le moins exagérée ou partiale. Il est certain que l'école primaire, bien qu'elle ait été fréquemment le terrain privilégié de l'expérimentation pédagogique, est tentée plus qu'elle ne devrait par l'acceptation passive des nouveautés et le maintien implicite de ses habitudes pédagogiques traditionnelles. Cela a été souligné tout au long de cet article par quelques exemples. Mais malgré tout, l'école primaire, les maîtres, les familles et bien sûr, les enfants n'ont pas été étrangers à l'évolution sociale et culturelle de notre époque (Dussel, 2005). L'enseignement primaire a changé, mais sans doute pas au rythme et de la façon dont le souhaiterait la pédagogie la plus avancée ou dont une société comme celle d'aujourd'hui a besoin.

C'est pourquoi le préjugé si répandu selon lequel l'école primaire, comparée au secondaire, est une oasis paisible et stable et un espace éducatif consolidé, avec un profil pédagogique bien défini, est tout aussi faux et partial, mais surtout injuste et vain. On sait que le taux d'échec dans l'enseignement secondaire obligatoire est encore élevé en Espagne ( $25 \%$ des élèves n'obtiennent pas le certificat de diplômé du secondaire), mais on sait aussi qu'un pourcentage semblable d'élèves du primaire terminent cette étape avec un niveau insuffisant pour les apprentissages instrumentaux et que leurs problèmes de comportement sont beaucoup plus nombreux qu'on ne l'imagine.

La prise en compte de l'échec et de l'inadaptation au primaire devrait donc être une priorité qu'il n'est possible de développer qu'en concevant l'école 
comme un espace communautaire. Il est absurde d'élaborer des programmes de compensation et de diversification curriculaire avec une orientation strictement académique et institutionnelle, ou trop tardivement, alors que l'élève est en grave danger d'exclusion sociale. L'école primaire a besoin d'une évaluation systématique de tous les facteurs et acteurs qui en font partie, afin d'améliorer sa qualité et son degré d'équité. L'évaluation doit fournir une information fiable sur l'acquisition de compétences, mais également mettre en garde contre les foyers potentiels d'inégalité et de discrimination. Cependant, il faut avertir du danger que court l'école primaire publique si elle est touchée de plein fouet par les évaluations standardisées, et autres avatars mercantiles, comme ceux qui envahissent déjà d'autres étapes du système scolaire. Elle y perdrait probablement quelque chose d'essentiel, son sens civique.

L'enseignement primaire, loin d'être une étape éducative aboutie et stable, a encore de nombreuses zones d'ombre et affronte de nouvelles perspectives dont le destin est incertain. C'est le cas par exemple de sa nécessaire adaptation à un cadre politique et administratif largement décentralisé qui, dans le même temps, participe au projet européen et assiste à l'accroissement accéléré de la diversité culturelle en raison des flux migratoires. Cette école n'a plus rien à voir avec celle d'il y a à peine vingt ans.

L'enseignement primaire a toujours un passif sur le plan pédagogique. La lenteur avec laquelle les écoles primaires acceptent certaines innovations pédagogiques et leur attachement excessif à des formules désormais caduques sont toujours préoccupants. Mais l'essentiel est que l'enseignement primaire retrouve sa raison d'être comme pivot de la scolarité de base et comme véritable socle du système scolaire. Il doit pour cela cesser d'être une simple étape préparatoire au second degré et retrouver son autonomie en tant qu'espace éducatif différencié, avec des objectifs, un style pédagogique et un curriculum propres, tout en améliorant la transition avec l'étape pré-élémentaire et le secondaire. Que doit enseigner l'école primaire et comment doit-elle s'organiser pour le faire efficacement, sont, aujourd'hui plus que jamais, des questions cruciales. La professeure argentine Inés Dusell (2005) propose une excellente idée, celles des "nouvelles alphabétisations", ce qui va bien au-delà du simple maniement des nouvelles technologies et touche l'ensemble de l'expérience scolaire.

\section{BibliographiE}

BOLÍVAR Antonio et RODRIGUEZ DIÉGUEZ José Luis (2002) : Reformas y retórica. La reforma educativa de la LOGSE. Málaga, Aljibe.

DE PUELLES BENÍTEZ Manuel (2005) : «Ocho leyes orgánicas en 25 años ». Cuadernos de Pedagogía, 348 : 12-14. 
DOMÉNECH Joan (2005) : «Los cambios en la Educación Primaria». Cuadernos de Pedagogía, 348 : 80-83.

DUSSEL Inés (2005) : «De la Primaria a la EGB : ¿qué cambió en la enseñanza elemental en los últimos años?». Buenos Aires, Facultad Latinoamericana de Ciencias Sociales (Área de Educación). Texte en cours de publication.

FEITO Rafael (2005) : «LOE : límites y logros del talante socialdemócrata». Cuadernos de Pedagogía, 347 : 82-86.

GONZÁLEZ FARACO Juan C. (1999) : «El currículum atrofiado : del pensamiento innovador a la práctica docente. Estudio longitudinal de los Proyectos de Educación Ambiental en Andalucía». Revista Española de Pedagogía, 57 (213) : 369-388.

GREEN Andy, LENEY Tom, et WOLF Alison (2001) : Convergencias y divergencias en los sistemas europeos de educación y formación profesional. Barcelona, Pomares.

LLEDÓ Angel (1999) : «¿Qué pasa con la Educación Primaria?». Cuadernos de Pedagogía, $280: 83-88$.

MINISTERIO DE EDUCACIÓN Y CIENCIA (1998) : Elementos para un diagnóstico del Sistema Educativo español. Informe Global. Madrid : Instituto Nacional de Calidad y Evaluación.

MINISTERIO DE EDUCACIÓN Y CIENCIA (2001) : Evaluación de la Educación Primaria 1999. Madrid, Instituto Nacional de Calidad y Evaluación.

PÉREZ DÍAZ Víctor y RODRÍGUEZ Juan C. (2003) : La educación general en España. Madrid, Fundación Santillana.

PEREYRA Miguel, GONZÁLEZ FARACO Juan Carlos et TORRES Mónica (2005) : «Cambio político, escuela y exclusión social : la última reforma escolar española del siglo XX relatada por sus actores", dans LUENGO, Julián (comp.) : Paradigmas de gobernación y exclusión social en la educación. Barcelona-México, Pomares, pp. 199-239.

RAMBLA Xavier et BONAL Xavier (2000) : «La política educativa y la estructura social», dans ADELANTADO José (eds.) Cambios en el Estado de Bienestar. Políticas sociales y desigualdades en España. Barcelona, Icaria-UAB, pp. 285-309.

RAMÍREZ Francisco et VENTRESCA Marc (1992) : «Institucionalización de la escolarización masiva : isomorfismo ideológico y organizativo en el mundo moderno ». Revista de Educación, 298 : 121-139. 
ROGERO Julio (1997) : «Cambios y resistencias al cambio en la Educación Primaria». Signos : Teoría y práctica de la educación, 22 : 34-43.

SEVILLA Diego (2003) : "La educación comprensiva en España : paradoja, retórica y limitaciones ». Revista de Educación, 330 : 35-58.

VIÑAO Antonio (1992) : «La Educación General Básica. Entre la realidad y el mito ». Revista de Educación ( ${ }^{\circ}$ extra. : La Ley General de Educación, veinte años después), pp. 47-71. 